\title{
TOPOLOGY OF QUATERNIONIC MANIFOLDS
}

\author{
BY VIVIAN YOH KRAINES ${ }^{1}$
}

Communicated by S. S. Chern, December 30, 1964

We give here a quaternionic analogue (Theorem 4) of the Hodge decomposition theorem [2, p. 26] for a Riemannian manifold with holonomy group contained in $\mathrm{Sp}(n) \times \mathrm{Sp}(1)$. Applying Chern's theorem in [1] (also [3]), we obtain some consequences on Betti numbers (Theorem 5).

Let $K^{n}$ denote the $n$-dimensional vector space over the field $K$ of quaternions, with the inner product

$$
(p, q)=\frac{1}{2} \sum_{i=1}^{n}\left(p_{i} \bar{q}_{i}+q_{i} \bar{p}_{i}\right)
$$

where

$$
\begin{aligned}
p & =\left(p_{1}, \cdots, p_{n}\right), \quad q=\left(q_{1}, \cdots, q_{n}\right) \text { and } \\
p_{i} & =p_{i}^{0}+p_{i}^{1} i+p_{i}^{2} j+p_{i}^{3} k \\
q_{i} & =q_{i}^{0}+q_{i}^{1} i+q_{i}^{2} j+q_{i}^{3} k
\end{aligned}
$$

are quaternions.

Let $\operatorname{Sp}(n)$ be the set of all endomorphisms, $A$, of $K^{n}$, satisfying the identity $(A \boldsymbol{p}, A \boldsymbol{q})=(\boldsymbol{p}, \boldsymbol{q}) . \mathrm{Sp}(n)$ is the set of all $n \times n$ matrices preserving the inner product. Then $\mathrm{Sp}(1)$ is the set of all unit quaternions. We define the action of $\mathrm{Sp}(n) \times \mathrm{Sp}(1)$ on $K^{n}$ as follows:

$$
(A, \lambda) p=A p \lambda, \quad \text { for }(A, \lambda) \in \mathrm{Sp}(n) \times \mathrm{Sp}(1),
$$

i.e., we multiply $p$ on the left by the matrix $A$ and on the right by the unit quaternion $\lambda$.

Definition. We define three skew symmetric bilinear forms $\Omega_{I}, \Omega_{J}$ and $\Omega_{K}$ on $K^{n}$ as follows:

$$
\begin{aligned}
& \Omega_{I}(p, q)=(p i, q), \\
& \Omega_{J}(p, q)=(p j, q) \text { and } \\
& \Omega_{K}(p, q)=(p k, q) .
\end{aligned}
$$

Note that $\Omega_{I}, \Omega_{J}$ and $\Omega_{K}$ may be thought of as exterior 2 -forms of $K^{n}$ considered as a $4 n$-dimensional real vector space.

1 This research is supported by NSF Contract No. GP-1610. The author wishes to express her gratitude to Professor Shoshichi Kobayashi. 
Definition. We define an exterior 4 -form $\Omega$ on $K^{n}$ by

$$
\Omega=\Omega_{I} \wedge \Omega_{I}+\Omega_{J} \wedge \Omega_{J}+\Omega_{K} \wedge \Omega_{K}
$$

THEOREM 1. $\Omega$ is invariant under the action of $\operatorname{Sp}(n) \times \operatorname{Sp}(1)$.

THEOREM 2. $\Omega^{n}=\Omega \wedge \Omega \wedge \cdots \wedge \Omega(n$ times $) \neq 0$.

Definition. A $4 n$-dimensional Riemannian manifold $M$ is called a quaternionic manifold if its holonomy group is a subgroup of $\operatorname{Sp}(n)$ $\times \operatorname{Sp}(1)$.

If $M$ is a quaternionic manifold of dimension $4 n$, then, by Theorems 1 and 2, we have a differential 4-form $\Omega$ on $M$ of maximal rank (i.e., $\Omega^{n} \neq 0$ ) which is parallel. Hence, $\Omega$ is a harmonic form. From the fact that $\Omega^{n} \neq 0$, we have

THEOREM 3. If $B_{i}$ denotes the ith Betti number of a quaternionic manifold $M$ of dimension $4 n$, then we have $B_{4 i} \neq 0$ for $i=0,1, \cdots, n$.

We define the operator ${ }^{*}$ which sends a $p$-form into a $(4 n-p)$-form in the usual way.

Definition. Define two operators $L$ and $\Lambda$ on the differential forms by

$$
L w=\Omega \wedge w, \quad \Lambda w=*(\Omega \wedge * w) .
$$

A differential form $w$ is called effective if $\Lambda w=0$.

Theorem 4. Let w be a p-form; then

$$
w=w_{e}^{p}+L w_{e}^{p-4}+\cdots+L^{r} w_{e}^{p-4 r}, \text { for } p \leqq n,
$$

where $w_{e}^{\boldsymbol{k}}$ is an effective $k$-form, and $r=[p / 4]$.

From Theorem 4 , it follows that $L$ sending $p$-forms into $(p+4)$ forms is $1-1$ for $p \leqq n-4$.

THEOREM 5. We have an increasing sequence of Betti numbers,

$$
\begin{gathered}
B_{i} \leqq B_{i+4} \leqq \cdots \leqq \\
\text { BIBLIOGRAPHY }
\end{gathered}
$$

1. S. S. Chern, On a generalization of Kähler geometry, Algebraic geometry and topology. A Symposium in honor of S. Lefschetz, pp. 103-121, Princeton Univ. Press, Princeton, N. J., 1957.

2. A. Weil, Introduction à l'étude des variêtés Kähleriennes, Hermann, Paris, 1958.

3. - Thêrème fondamental de Chern en géométrie riemannienne, Séminaire Bourbaki, 14e année, 1961/62, no. 239.

University of California, Berkeley 